\title{
Multi-Body Kinematics of Shovel Crawler Performance in Rugged Terrains
}

\author{
${ }^{1}$ Samuel Frimpong, ${ }^{2}$ Magesh Thiruvengadam \\ ${ }^{I}$ Professor and Robert H. Quenon Endowed Chair, Missouri University of Science and Technology, Rolla, USA. \\ ${ }^{2}$ Research Assistant Professor, Missouri University of Science and Technology, Rolla, USA.
}

*Corresponding Author: Samuel Frimpong, Professor and Robert H. Quenon Endowed Chair, Missouri University of Science and Technology, Rolla, USA.

\begin{abstract}
Electric rope shovels are widely used in surface mining operations due to their large production capacities. These shovels use crawler tracks for terrain engagement. Shovel reliability, maintainability and availability rely on track service life. Fatigue failure of crawler tracks, in rugged and abrasive geological formations, occurs extensively causing high maintenance costs and production losses. This study investigates the crawler-formation interaction in tough and rugged terrains of oil sands for understanding crawler failure problems. The governing equations of crawler-formation interaction kinematics have been formulated for link pin joint, oil sands joint and driving constraints to capture crawler motion during shovel production based on the rigid multi-body theory. The crawler is powered with translation and rotation driving constraints to produce driving and turning propel motion son the rugged oil sands terrain.

The kinematic modeling of a 3-D crawler-terrain interaction shows that the crawler motion on oil sands terrain develops 132 degrees of freedom (DOFs) and dynamic modeling is required to calculate those DOFs. A 3-D virtual prototype model of the crawler-formation interactionis builtin MSC. ADAMS based on the rigid-body kinematics to simulate the crawler propel motion for given driving constraints. The results from the driving constraints yield a non-linear longitudinal motion of the crawler track assembly. The track's lateral and vertical displacements during translation fluctuate between 0.7 and $3.6 \mathrm{~cm}$. The maximum longitudinal, lateral and vertical velocities are $0.22,0.046$ and $0.56 \mathrm{~m} / \mathrm{s}$, while the maximum accelerations along longitudinal, vertical and lateral directions are 7.41, 34.9 and $1.73 \mathrm{~m} / \mathrm{s} 2$, respectively. This research forms the basis for modeling rigid-flexi blevirtual simulator of the crawler-terrain interaction for predicting crawler fatigue life.
\end{abstract}

Keywords: Rigid multi-body system, shovel crawler kinematics, flexible oil sands terrain, track-ground interaction, virtual prototype simulation

\section{INTRODUCTION}

Cable shovels are widely used in surface mining operations. The shovel consists of three major assemblies - the lower works or undercarriage, upper body and the front-end assembly. The lower works, which support the upper body and attachment assemblies of cable shovel, consists of propel and crawler systems as shown in Figure 1. The shovel load due to weight of the upper body, attachments and dipper payload acting on the lower works model in Figure 1 are established using load data of P\&H 4100C BOSS Electric Shovel $[1,2]$. The crawler system is made up of crawler tracks, idlers, rollers and guide rails [3]. The tracks consist of crawler shoes that are connected together by link pins to form a continuous chain [3]. During propel or loading, the weight of the shovel, front-end assembly and the dipper payload are transmitted to the terrain in Figure 1. Under abrasive and rugged terrains, such as in oil sands, the crawler shoes can wear and tear causing premature shoe failure [3]. The multi-body kinematics study on crawler - terrain interactions in surface mining is non-existent. However, some researchers have carried out track-terrain interaction studies on military tracked vehicles and on hydraulic excavators [4-6].The purpose of this chapter is to simulate, under virtual field conditions, the3-D shovel crawler - terrain interaction kinematics using MSC.ADAMS and determine position, velocity and accelerations of each shoe for two types of shovel propel motions. The motions include translation only, and both translation and rotational. The results of this study form the foundations for the detailed crawler-terrain dynamics, fatigue and lifecycle modeling and analysis of shovel crawler assembly. 


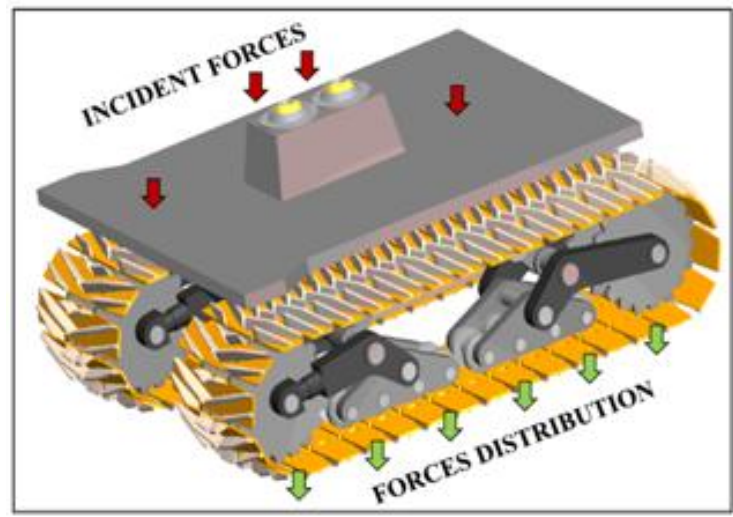

Figure1. Shovel crawler-formation interactions [7]

\section{Rigid MULTI-BODY KinEMATICS OF CRAWLER-TERRAIN INTERACTIONS}

Figure 1 shows the schematic of crawler track-terrain interactions. In this study, only the open track chain (part of the track assembly) in contact with the ground is modeled using dimensions of the P\&H 4100C Boss shovel in Table 1. Since the crawler track assembly consists of crawler shoes, a crawler shoe geometry based on the actual shoe model for the P\&H 4100C Boss shovel [1, 2]is developed first as shown in Figure 2. The crawler shoes are then linked to development-body system of the crawler track assembly shown in Figure 3.

Table1. Crawler dimensions of P\&H 4100C Electric Shovel [1]

\begin{tabular}{|l|l|}
\hline Width of Crawler Shoes & $3.505 \mathrm{~m}$ \\
\hline Width of Crawlers & $12.8 \mathrm{~m}$ \\
\hline Length of Crawlers & $11.6 \mathrm{~m}$ \\
\hline
\end{tabular}

The link pins used to connect the crawler shoes to form a continuous track are not included in the multi-body model but considered through joint constraints equations. The rest of the crawler system parts, such as idlers, rollers, drive tumbler, and guide rails does not have joint connection but only surface or line contact with the track. The contact forces from the interactions among the track and other crawler system components can be ignored since the crawler shoe fatigue failure mainly occurs due to the track-formation interaction and the distribution of shovel weight and dipper payload on the crawler shoes [3]. However, the weight of the attachments (dipper, boom, and crowd), carbody with swing gear and tapered rollers, propel system, crawler system (rollers, driver tumblers, guide rails, idlers, link pin and crawler frame) and dipper payload are applied as external loads on the crawler tracks for dynamic analysis.

The oil sands terrain is modeled using 50 oil sands units attached to the ground link using a springmass-damper system [8]. The multi-body crawler track model, consisting of 13 crawler shoes interacting with flexible oil sands terrain and made up of 50 oil sands units, results in a total number of bodies, $n_{c}=63$, and is shown in Figure 3. The oil sand terrain has total length, width and depth of $70 \mathrm{~m}, 35 \mathrm{~m}$ and $2 \mathrm{~m}$, respectively. The body numbers for each crawler shoe and oil sand unit are also shown in Figure 3. The density and mass of crawler shoe material and oil sand units used in this research study is shown in Table 2 from [8].

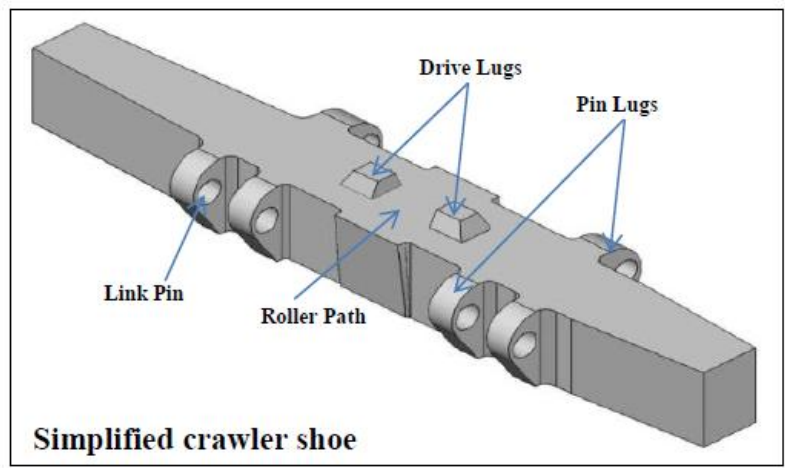

Figure2. Isometric view of a crawler shoe [2] 


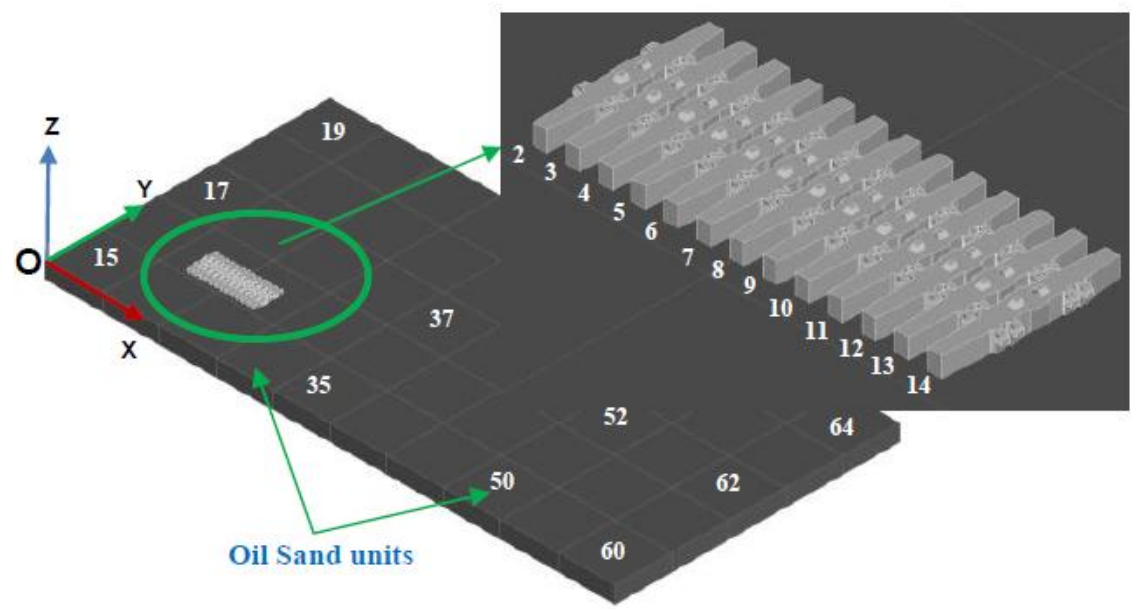

Figure3. Crawler track assembly interacting with the ground

The 3-D virtual multi-body prototype model (in Figure 3) is built in MSC.ADAMS to simulate the crawler-terrain interaction kinematics. In MSC. ADAMS, body-fixed 313 Euler angles $(\psi, \theta, \phi)$ are used to establish angular orientation of the body relative to global coordinate system (GCS) $\mathrm{O}$ in Figure 3[9]. Also in MSC.ADAMS, mass and mass moments of inertia of each body are used both in the kinematics and dynamic analysis of the crawler assembly [9]. If the system DOF is zero, MSC.ADAMS solves the joint and motion constraint equations to calculate kinematic variables for each time step and uses dynamic equations of motion to calculate reaction forces. If the system DOF is greater than zero, MSC.ADAMS solves the differential algebraic equations (DAEs) consisting of kinematic constraint equations and dynamic equations of motion with external and constraint forces using GSTIFF integrator to obtain crawler kinematics and constraint forces $[9,10]$.

Table2. Mass Properties of System

\begin{tabular}{|c|c|c|c|}
\hline Body & Density $\left.\mathbf{( k g} / \mathbf{m}^{\mathbf{3}}\right)$ & Volume $\left.\mathbf{( m}^{\mathbf{3}}\right)$ & Mass $(\mathbf{k g})$ \\
\hline Crawler Shoe & 7847.25 & 0.5966 & 4681.67 \\
\hline Oil-sand unit & 1600.0 & 98.0 & $1.568 \times 10^{5}$ \\
\hline
\end{tabular}

Shovel Crawler Kinematic Equations: All the kinematic equations are developed based on Shabana $[11,12]$ for multi-body systems. The crawler shoes are assumed to be rigid. From Shabana [11], the configuration of each component $i$ in the system is identified using the absolute Cartesian generalized coordinates vector $\mathbf{u}_{i}$. The generalized coordinates of the system in vector form is given by Shabana [11] as equations (1) - (3).

$$
\begin{aligned}
& \mathbf{u}_{i}=\left[\begin{array}{ll}
\mathbf{B}_{i}{ }^{T} & \gamma_{i}{ }^{T}
\end{array}\right]^{T} \\
& \mathbf{B}_{i}=\left[B_{x_{i}}, B_{y_{i}}, B_{z_{i}}\right]^{T} \text { and } \boldsymbol{\gamma}_{i}=\left[\psi_{i}, \theta_{i}, \phi_{i}\right]^{T}
\end{aligned}
$$

$i=2,3, \ldots \ldots, 64$ denote body number; $\boldsymbol{B}_{\boldsymbol{i}}{ }^{-}$global position vector of body $i$; and $\boldsymbol{\gamma}_{\boldsymbol{i}^{-}}$-set of independent Euler angles of body $i$. The number of generalized coordinates inthe multi-body system shown in Figure 3 is obtained from equation (1) as $n=6 \times 63=378$.

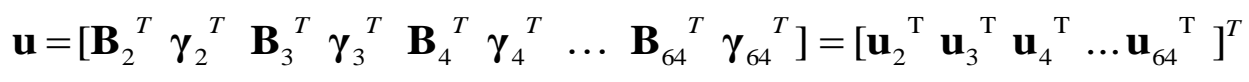

The generalized coordinates in equation (3) are not independent because of the joint and driving constraints [11]. The kinematic constraints equations that describe the joints and motion can be given as equation (4).

$$
\mathbf{K}(\mathbf{u}, t)=\left[\begin{array}{llll}
K_{1}(\mathbf{u}, t) & K_{2}(\mathbf{u}, t) & K_{3}(\mathbf{u}, t) & \cdots \cdots \cdot K_{n_{k}}(\mathbf{u}, t)
\end{array}\right]^{\mathrm{T}}=0
$$

$\mathbf{K}$ is a vector of constraint functions; $n_{k}$ is the total number of system constraint equations; and $t$ is time. The kinematic constraint velocity equation is given as equation (5) by differentiating equation (4) with respect to $t$.

$$
\mathbf{K}_{\mathbf{u}} \dot{\mathbf{u}}=-\mathbf{K}_{t}
$$


Similarly, by differentiating equation (5) with respect to $t$, the kinematic constraint acceleration equations can be generated as equations (6) - (10).

$\mathbf{K}_{\mathbf{u}} \ddot{\mathbf{u}}=\mathbf{E}$

$\mathbf{E}=-\left(\mathbf{K}_{\mathbf{u}} \dot{\mathbf{u}}\right)_{\mathbf{u}} \dot{\mathbf{u}}-2 \mathbf{K}_{\mathbf{u} t} \dot{\mathbf{u}}-\mathbf{K}_{t t}$

$\dot{\mathbf{u}}_{i}=\left[\begin{array}{llllll}\dot{B}_{x_{i}} & \dot{B}_{y_{i}} & \dot{B}_{z_{i}} & \dot{\psi}_{i} & \dot{\theta}_{i} & \dot{\phi}_{i}\end{array}\right]^{T}-$ vector of generalized velocity of body $i$

$\ddot{\mathbf{u}}_{i}=\left[\begin{array}{llllll}\ddot{B}_{x_{i}} & \ddot{B}_{y_{i}} & \ddot{B}_{z_{i}} & \ddot{\psi}_{i} & \ddot{\theta}_{i} & \ddot{\phi}_{i}\end{array}\right]^{T}-$ vector of generalized acceleration of body $i$

$\mathbf{K}_{\mathbf{u}}=\frac{\partial \mathbf{K}}{\partial \mathbf{u}}-$ Jacobian of the constraint equation (4)

$\mathbf{K}_{t}$ and $\mathbf{K}_{t t}$ - partial derivatives of constraint function vector $\mathbf{K}$ with respect to $t ; \mathbf{K}_{\mathbf{u} t}-$ partial derivative of constraint vector Jacobi an $\mathbf{K}_{\boldsymbol{u}}$ with respect to $t$. The kinematic constraint equations for the crawler and oil sands in Figure 3 are described in the following paragraphs.

Revolute Joint Constraints: The crawler shoes are connected to each other using two link pins in an actual crawler assembly(Figure 4). The link pins rotate about the axis of rotation as in Figure 4. This rotation reduces loading in the shoe lugs and increases the life of the link pin [3]. This link pin constraintis called a revolute joint and has one rotational DOF [11, 13]. Researchers [4-6, 14-19] used revolute or pin joints for their analysis. Many of these studies simulated the connection between the track links for slow moving tracked vehicle using revolute joints with one DOF. Rubinstein and Hitron[6] and Madsen [15] added friction to the revolute joint to simulate the contact forces between pin and hole. This study neglects the effect of friction and treats the crawler shoe links as frictionless one DOF revolute joint. Since two link pin joint axis is collinear (Figure 4), revolute joints for two link pins to connect two rigid crawler shoes result in redundant constraints. Redundant constraints can be eliminated, if one pin joint is made a parallel primitive joint and the other pin joint is a spherical joint $[9,10]$.

Spherical Joint Constraints: For spherical joints in Figure 5, the points $\mathrm{P}_{\mathrm{i}}$ and $\mathrm{P}_{\mathrm{j}}$ on bodies $i$ and $j$ always coincide, thus removing three relative translations along the joint $x, y$ and $z$ axes [11]. The three constraint equations for spherical joints from Shabana [11] can be derived from equation (11).

$\mathbf{K}_{S}\left(\mathbf{u}_{i}, \mathbf{u}_{j}\right)=\mathbf{r}_{P_{i}}-\mathbf{r}_{P_{j}}=\mathbf{0}$

$i=2,3, \ldots, 13$ and $j=3,4, \ldots, 14$ and $\mathbf{r}_{P_{i}}, \mathbf{r}_{P_{j}}$ are global position of point $\mathrm{P}$ on body $i$ and body $j$. Equation (11) can be rewritten in terms of generalized coordinates of bodies $i$ and $j$ defined by equation (12).

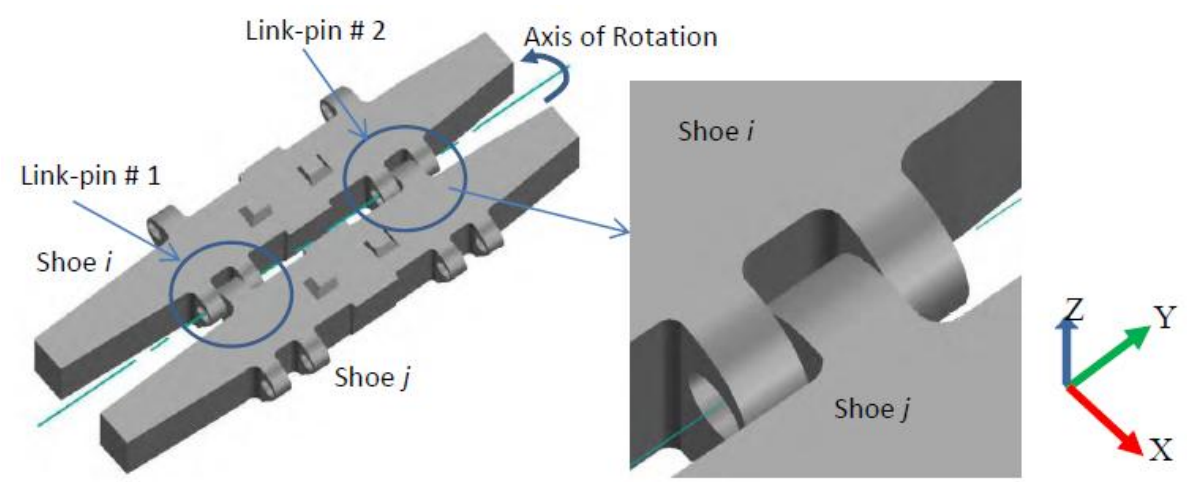

Figure4. Crawler shoe link pin joints

$\mathbf{K}_{S}\left(\mathbf{u}_{i}, \mathbf{u}_{j}\right)=\mathbf{r}_{P_{i}}-\mathbf{r}_{P_{j}}=\mathbf{B}_{i}+\mathbf{A}_{i} \mathbf{s}_{P_{i}}^{\prime}-\mathbf{B}_{j}-\mathbf{A}_{j} \mathbf{s}_{P_{j}}^{\prime}=\mathbf{0}$

$\mathbf{A}_{i}$ and $\mathbf{A}_{j}$ are the rotation matrices that define the orientation of the body $i$ and $j$ with respect to the global coordinate system [11], and $\mathbf{s}_{P_{i}}^{\prime}$ and $\mathbf{s}_{P_{j}}^{\prime}$ are the positions of the point $\mathrm{P}$ on body $i$ and body $j$ with respect to its centroidal coordinate system. 


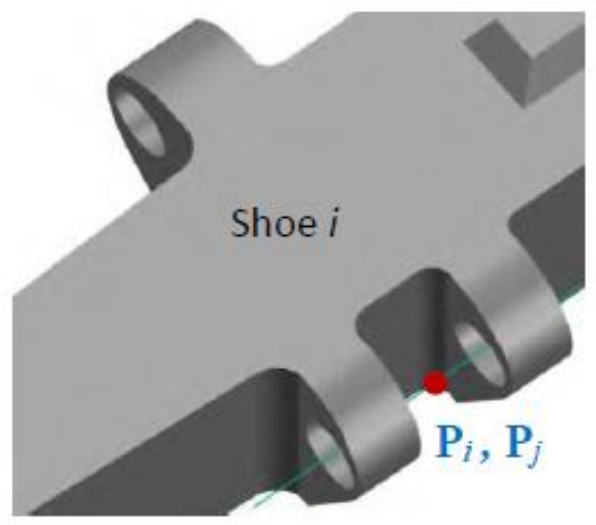

Figure5. Bodies $i$ and $j$ connected by spherical joint

Parallel Primitive Joint Constraints: The bodies, labelled $2-14$, are connected by parallel primitive joint at the second link pin location in Figure 4. In Figure 6, the vectors $\mathbf{a}_{i}, \mathbf{b}_{i}$ and $\mathbf{c}_{i}$ and vectors $\mathbf{a}_{j}, \mathbf{b}_{j}$ and $\mathbf{c}_{j}$ are defined along the joint $x, y$ and $z$ coordinate axes defined for bodies $i$ and $j$ at the joint location $\mathrm{P}[9,11]$. The two constraint equations for parallel primitive joints are from equation (13).
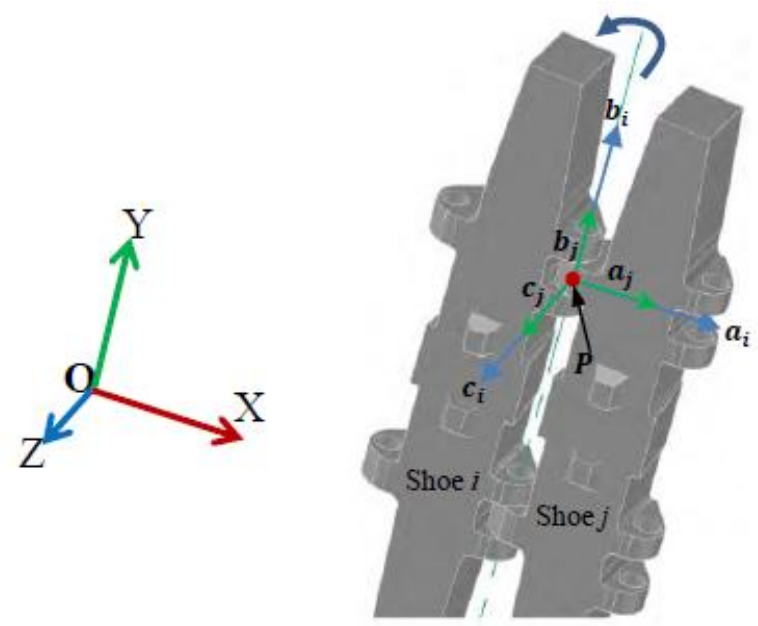

Figure6. Bodies $i$ and $j$ connected by primitive joint

$\mathbf{K}_{P}\left(\mathbf{u}_{i}, \mathbf{u}_{j}\right)=\left[\begin{array}{l}\mathbf{c}_{i} \bullet \mathbf{b}_{j} \\ \mathbf{a}_{i} \bullet \mathbf{b}_{j}\end{array}\right]=\mathbf{0}$

$i$ and $j=2,3,4, \ldots, 13$ and $3,4,5, \ldots, 14$

Driving Constraints: Figure 7 shows the two types of motion constraints used in this study to propel the crawler track on the oil sands terrain. The motion constraints are applied at the center of mass of Part 14 (shoe 13) (point P14) in Figure 7. In the first motion type, the track undergoes translation motion. In the second motion type, the crawler track undergoes both translation and rotational constraint to complete a turning motion. The kinematic constraint equation for both translation and turning motions is described below.

Translation Driving Constraints: The translation driving constraint is given as equation (14).This constraint translates the crawler assembly with a velocity $v_{x}(t)$ varying as a cubic function of time $(\mathrm{t})$ in the global positive $\mathrm{x}$-direction. The crawler is free to move in the remaining two translation and three rotation DOFs. The non-linear cubic velocity function on shoe 13 may cause large variations in the displacement, velocity and acceleration components of different shoes which in turn may exert maximum loadings and impact crawler shoe fatigue life.

$$
K_{T D}\left(\mathbf{u}_{14}, t\right)=B_{x_{14}}-B_{0, x_{14}}-\int v_{x}(t) \mathrm{d} t=0
$$


$B_{0, x_{14}}=17.998 m$ is the initial global $x$-location of Part 14 (shoe 13) shown in Figure 3 at time $t=0$ and

$v_{x}(t)=\left[\begin{array}{lc}0.012 t^{2}-0.0016 t^{3} ; t_{1} \leq t \leq t_{2} \\ 0.1 & ; t>t_{2}\end{array}\right]$

The maximum velocity of a large mining shovel is reported by Ma and Perkins [14] as $0.25 \mathrm{~m} / \mathrm{s}$. This research limits the maximum input velocity of the crawler track to $0.1 \mathrm{~m} / \mathrm{s}$.
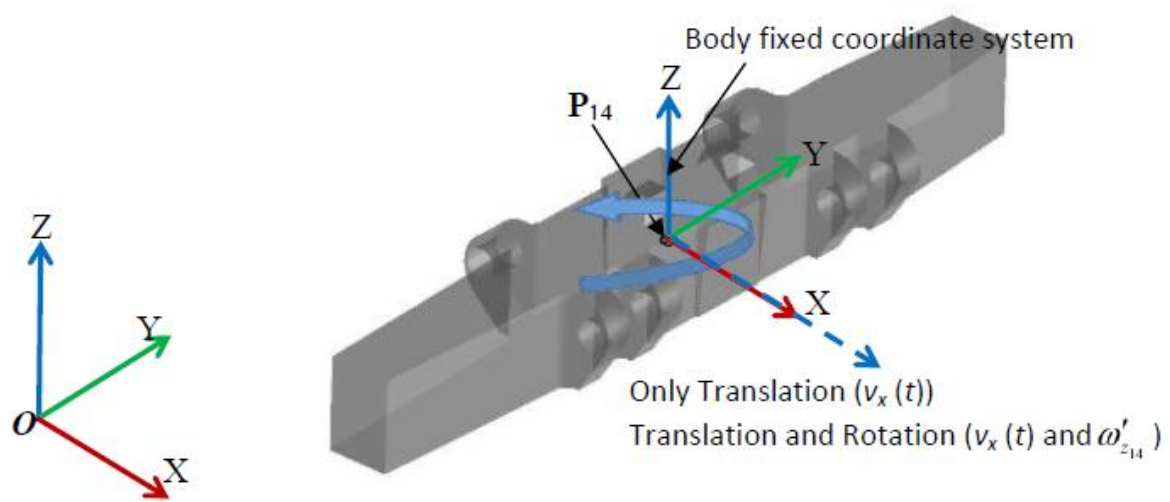

Figure7. Motion constraints on crawler shoe 13 (Part 14)

Translation and Rotation Driving Constraints: For turning motion, crawler shoe 13 is provided translation velocity $v_{x}(t)$ (equation (15)) and rotational velocity $\omega_{z_{14}}^{\prime}$ as shown in Figure 7. The translation driving constraintis same as equation (14)while the rotational constraint is non-holonomic and can only be expressed in terms of differential of coordinates and time as equation (16) from Shabana [12] and Schiehlen et al. [20].

$K_{R D}\left(\mathbf{u}_{14}, t\right)=\cos \theta_{14} * \dot{\psi}_{14}+\dot{\phi}_{14}-\omega_{z_{14}}^{\prime}=0$

$\omega_{z_{14}}^{\prime}$ is the angular velocity about the $z$-axis of body-fixed coordinate system and is defined in equation (17) similar to equation (15). $\psi_{14}, \theta_{14}$ and $\phi_{14}$ are rotation angles about the original $z$-, new $x$ - and new $z$-axes.

$\omega_{z_{14}}^{\prime}=\left[\begin{array}{ll}0.12 * t^{2}-0.016 * t^{3} & ; t_{1} \leq t \leq t_{2} \\ 1.0 & ; t>t_{2}\end{array}\right] \mathrm{deg} / s$

Oil Sands Constraints: Figure 3 shows that oil sands terrain is made of 50 oil sands units (bodies 1564). From Frimpong and Li [8] and Frimpong et al. [21],each oil sands unit has two translational and two rotational motion constraints at its center of mass that can be represented as a 2 DOFsspringmass-damper system and is connected to adjacent oil sands units by spherical joints as shown in Figures 8a and 8b. Two DOFs oil sands units connected by spherical joints results in redundant constraints and can be eliminated by removing two translation motion constraints on the oil sands units 1-49 (bodies 15-63) and by connecting adjacent oil sands units using combination of spherical and primitive in-plane joints as in Figure 9. The constraint equations for spherical joints connecting two adjacent oil sands units in Figure 9 are similar in form to equations (11) and (12). The constraint equation for in-plane joint can be expressed as in equation (18) [9].

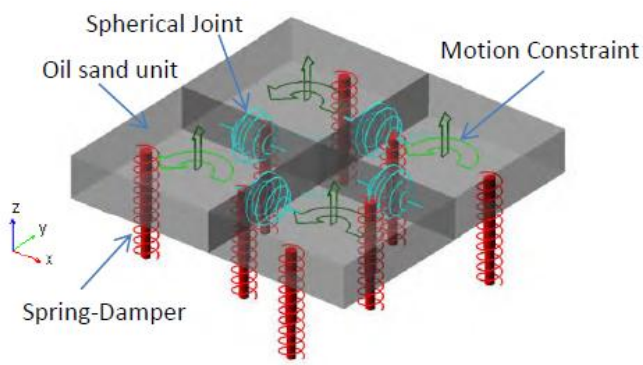

(a)

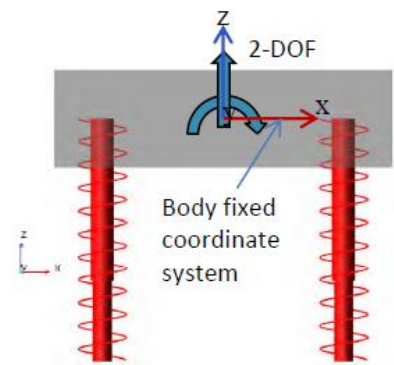

(b)

Figure8. Oil sand units with 2 DOFs connected by spherical joints [8, 21] 
$K_{I}\left(\mathbf{u}_{i}, \mathbf{u}_{j}\right)=r_{z_{i}}-r_{z_{j}}=0$

$r_{z_{i}}$ and $r_{z_{j}}$ are global z-coordinates of the in-plane joint location point on 2 adjacent oil sands units in Figure 9.

The two non-holonomic rotation motion constraint equations on oil sands units (bodies $i=15-64$ ) follow either equation (19) or (20) depending on the orientation of spring damper system shown in Figure 9.

$\mathbf{K}_{R}\left(\mathbf{u}_{i}\right)=\left[\begin{array}{l}\dot{\theta}_{i} \cos \psi_{i}+\dot{\phi}_{i} \sin \theta_{i} \sin \psi_{i} \\ \dot{\psi}_{i}+\dot{\phi}_{i} \cos \theta_{i}\end{array}\right]=\left[\begin{array}{l}\omega_{x_{i}} \\ \omega_{z_{i}}\end{array}\right]=\mathbf{0}$

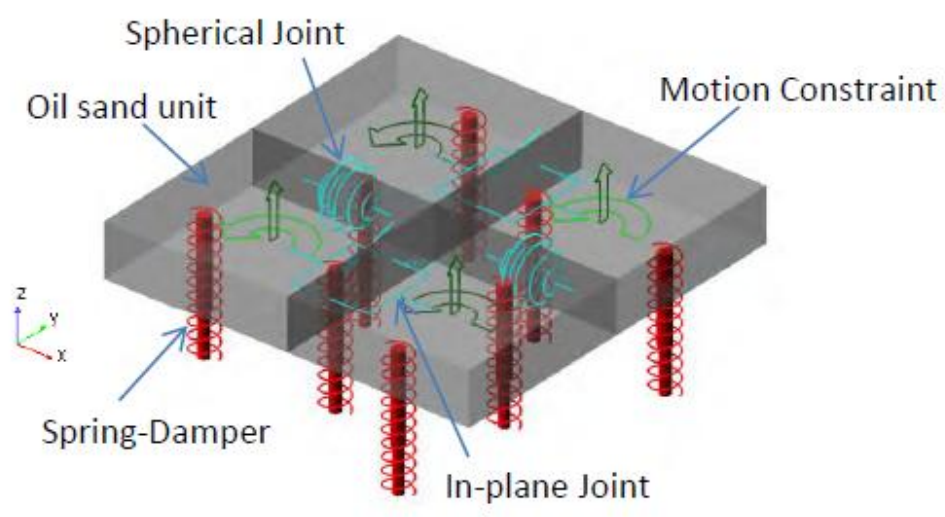

Figure9. Oil sand units with 2 DOFs connected by spherical and in-plane joints [8, 21]

$\mathbf{K}_{R}\left(\mathbf{u}_{i}\right)=\left[\begin{array}{l}\dot{\theta}_{i} \sin \psi_{i}-\dot{\phi}_{i} \sin \theta_{i} \cos \psi_{i} \\ \dot{\psi}_{i}+\dot{\phi}_{i} \cos \theta_{i}\end{array}\right]=\left[\begin{array}{l}\omega_{y_{i}} \\ \omega_{z_{i}}\end{array}\right]=\mathbf{0}$

$\omega_{x_{i}}, \omega_{y_{i}}$ and $\omega_{z_{i}}$ in equations (19) and (20) denote angular velocity of oil sands units about the global $x, y$ and $z$ axes, respectively. For oil sands unit 50 (Part 64), in addition to two rotational constraints expressed in equations (19) or (20),two translation constraints given by equation (21) are also provided.

$\mathbf{K}_{T}\left(\mathbf{u}_{64}\right)=\left[\begin{array}{l}B_{x_{64}}-B_{0, x_{64}} \\ B_{y_{64}}-B_{0, y_{64}}\end{array}\right]=\mathbf{0}$

$B_{0, x_{64}}=66.5 \mathrm{~m}$ and $B_{0, y_{64}}=31.5 \mathrm{~m}$ are the initial global $x$ and $y$ positions of oil sands unit 50 (Part 64) in Figure 3 at time $t=0$.In the multi-body system in Figure 3, there are(i) $n_{s}=36$ spherical joint constraint equations and $n_{p}=24$ parallel axes joint primitive constraintsequations due toconnection between crawler shoes; (ii) $n_{d}=1$ driving constraint equation due to crawler track translation motion; (iii) $n_{n d}=1$ non-holonomic driving constraint equation due to crawlertrack rotation motion; (iv) $n_{s o}=$ 147 spherical joint constraint equationsand $n_{i o}=36$ in-plane joint primitive constraint equations due to connection between oil sands units; (v) $n_{n o}=100$ non-holonomic equations due to rotation motion constraintson each oil sands unit; and (vi) $n_{m o}=2$ equations due to translation motion constrainton the last oil sands unit 50 or Part 64.

Hence the multi-body system has a total of $n_{k}=n_{s}+n_{p}+n_{d}+n_{n d}+n_{s o}+n_{i o}+n_{n, o}+n_{m, o}=$ 346 kinematic constraint equations for the translation only motion and $n_{k}=347$ for the turning motionresulting in the system generalized coordinates $(n=378)$ exceeding the total number of kinematic constraint equations $\left(n_{k}\right)$. Thiscondition, when $n_{k}$ is less than $n$, causes indeterminate kinematic system. The number of system DOF sin the presence of non-holonomic constraint equations can be calculated as $n-\left(n_{k}-n_{n d}-n_{n o}\right)=132$. Since system DOFs >zero, the differential algebraic equations consisting of dynamic equations of motion and kinematic constraint equations are required to be solved simultaneously using numerical methods to obtain displacement, velocity, and acceleration of each shoe during two types of propel motion. 
Shovel Crawler Dynamic Equations of Motion: The free body diagram of a crawler shoe $I$, with inertia forces in dynamic equilibrium with external and joint constraint forces, is shown in Figure 10 $[11,22]$. The external forces acting on the crawler shoe $i$ are the gravity force $\left(\mathrm{m}^{\mathrm{i}} \mathrm{g}\right)$ due to shoe weight, uniformly distributed load $\left(\mathrm{w}^{\mathrm{i}}\right)$ due to machine weight and contact forces $\left(\mathrm{F}_{\mathrm{C}}^{\mathrm{i}}, T_{\mathrm{C}}^{\mathrm{i}}\right)$ due to interaction between crawler shoe $i$ and ground as in Figure 10. The joint forces at the spherical joints $\left(\mathrm{F}_{\mathrm{S}}^{\mathrm{i}-1, \mathrm{i}}, T_{\mathrm{S}}^{\mathrm{i}-1, \mathrm{i}}\right.$ and $\left.\mathrm{F}_{\mathrm{S}}^{\mathrm{i}, \mathrm{i}+1}, T_{\mathrm{S}}^{\mathrm{i}, \mathrm{i}+1}\right)$ and parallel primitive joints $\left(\mathrm{F}_{\mathrm{P}}^{\mathrm{i}-1, \mathrm{i}}, T_{\mathrm{P}}^{\mathrm{i}-1, \mathrm{i}}\right.$ and $\left.\mathrm{F}_{\mathrm{P}}^{\mathrm{i}, \mathrm{i}+1}, T_{\mathrm{P}}^{\mathrm{i}, \mathrm{i}+1}\right)$ are also shown in Figure 10.The contact forces, and spring damper forces are the external forces acting on each oil sand unit $i$ as in Figure 11. The crawler shoes exert equal and opposite contact forces $\left(\mathbf{F}_{J}^{i}\right)$ and frictional torque ( $\mathbf{T}^{i}$ ) on oil sands unit $i$ at point $\mathbf{J}$ as in Figure 11.

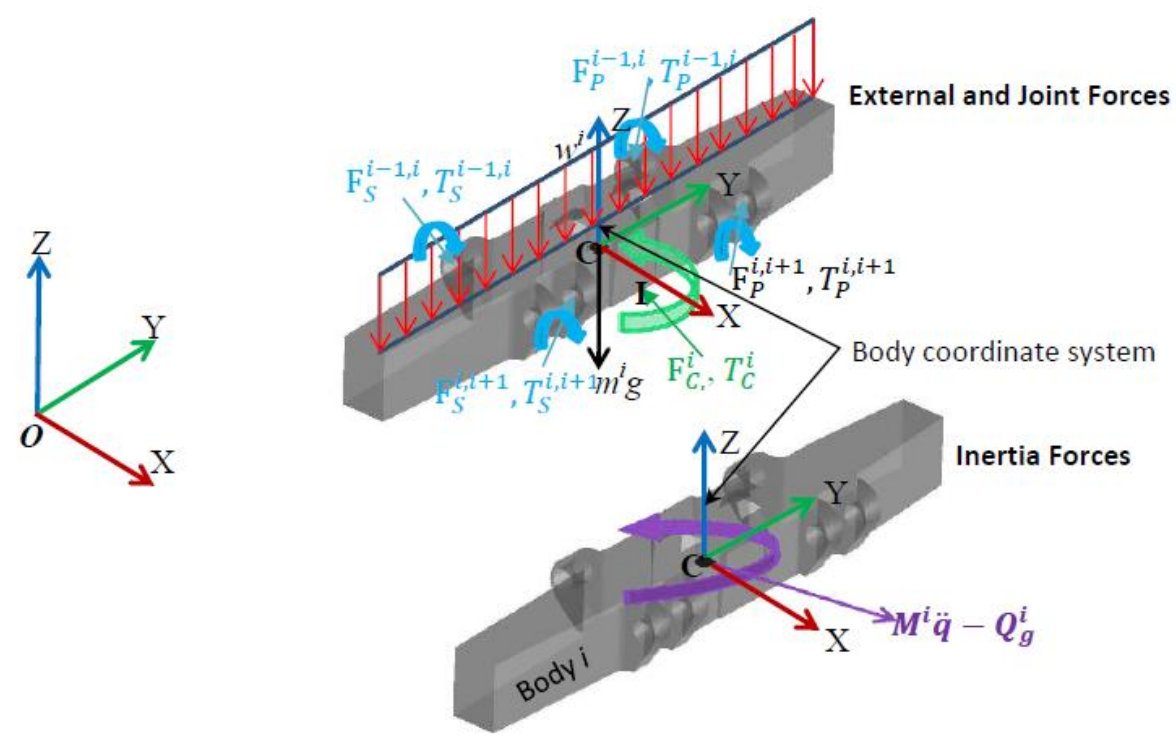

Figure10. Dynamic equilibrium of the rigid crawler shoe, $i$

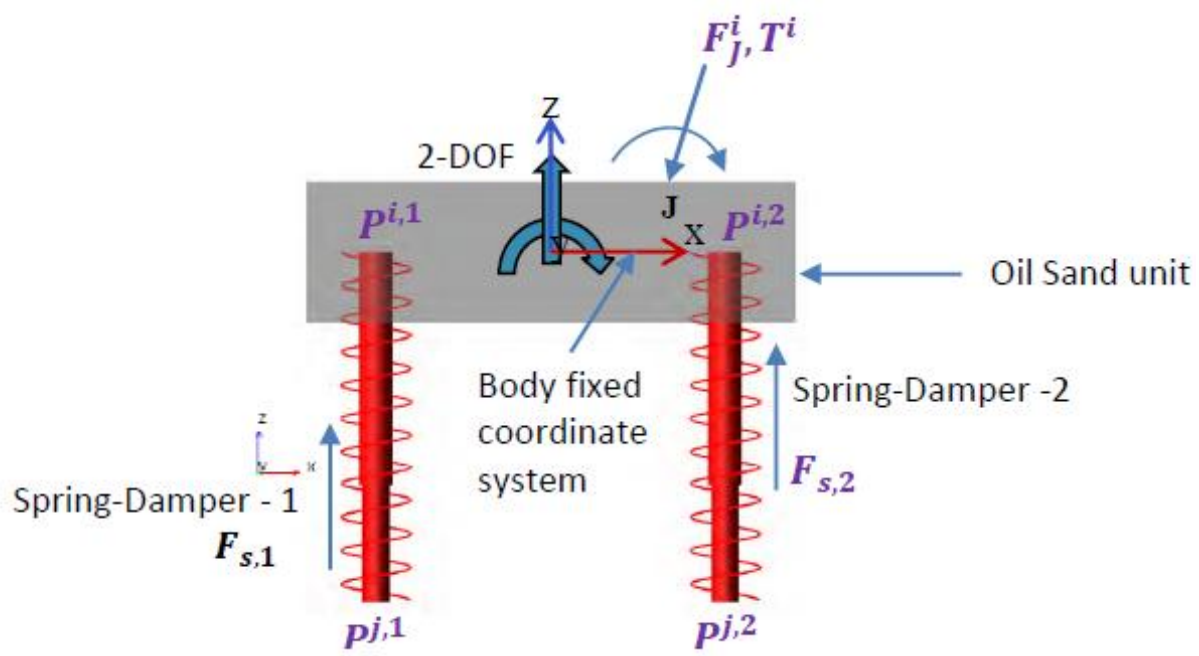

Figure11. External forces on oil sand unit $i$

In addition to the contact force, two spring-damper forces are also exerted on the oil sands unit as in Figure 11. This spring damper forces, $F_{s, 1}$ and $F_{s, 2}$, act along the line connecting points $P^{i, 1}$ and $P^{i, 2}$ on oil sands unit $i$ to corresponding points $P^{j, 1}$ and $P^{j, 2}$ on the ground link in MSC ADAMS (Figure 11). For $n_{c}=63$ interconnected rigid multi-body system in Figure 3, the differential equations of motion can be written from Shabana and $\operatorname{MSC}[9,11]$ as in equation (22).

$\mathbf{M u ̈}+\mathbf{K}_{\mathbf{u}}^{T} \boldsymbol{\lambda}=\mathbf{Q}_{\mathbf{a}}+\mathbf{Q}_{\mathbf{g}}$

$\mathbf{M}$ - Mass matrix of the system; $\mathbf{u}$ - vector of system generalized coordinates; $\mathbf{K}$ - constraint equations due to joints and applied motion; $\mathbf{Q}_{\mathrm{a}}$ - Applied forces; $\mathbf{Q}_{\mathrm{g}}$ - gyroscopic terms of the inertia 
forces. The total number of differential equations in equation (22) is $6 \times n_{c}=6 \times 63=378$, while the number of unknowns are the sum of $n=6 \times n_{c}=378$ generalized accelerations and $n_{k}=346$ or 347 Lagrange multipliers. From Shabana [11], the additional $n_{k}$ equations needed to solve for $n+n_{k}$ unknowns are obtained from the kinematic constraint equation (4).The system of differential algebraic equations (DAE) in equations (22) and (4)is solved numerically using MSC.ADAMS to predict the motion parameters and reaction forces.

Virtual Prototype Modeling and Simulation: A virtual prototype model of the crawler assembly in Figure 3 is built in MSC. ADAMS to solve the DAE equations (4) and (22) and to simulate the crawler-terrain interaction for capturing crawler kinematics during propel. The development of virtual prototype starts with building the crawler track and oil sands terrain assembly (Figure 3) in SOLIDWORKS and importing into MSC.ADAMS. The virtual prototype modeling is completed as follows: (i)the crawler shoes and oil sands units are assigned mass properties using inputs from Table 2; (ii)spring and damper elements with inputs from Table 3 are attached to each oil sands unit to define stiffness and damping characteristics of oil sands terrain;(iii) the spherical and primitive joints are used to establish connection between crawler shoes and between oil sands units; and (iii) the external forces due to gravity and machine load are defined on the crawler track. In addition the external forces arising from contact between crawler shoes and oil sands units are also defined.

The gravity force and distributed load on crawler shoes are constants and are entered directly as user input into MSC.ADAMS. The contact force, acting on each crawler shoe, is a time varying force and is calculated using the built-in contact force algorithm in MSC.ADAMS. These forces consist of normal and frictional forces and frictional torque. Figure 12 shows the contact forces $\left(F_{n}-\right.$ normal, $F_{t}-$ tangential and $\mathrm{T}$ - torque) between shoe $i$ and ground [22,23]. These forces act on the shoe bottom surface at a point I [9] as in Figure 12. The normal force vector $\mathbf{F}_{N}^{i}=\left[\begin{array}{lll}F_{N, x} & F_{N, y} & F_{N, z}\end{array}\right]^{T}$ acting at point 1 for shoe $i$ (Figure 12) is calculated using the impact function model in MSC.ADAMS. In this model, when two solid bodies come into contact with each other, a nonlinear spring damper system is used to determine the normal force $[9,10]$ as in equation (23).

$$
\mathbf{F}_{N}^{i}= \begin{cases}k x^{e}-c_{\text {max }} \dot{x}^{*} \operatorname{Step}(x, 0,0, d, 1) & \text { if } x>0 \\ 0 & \text { if } x \leq 0\end{cases}
$$

The coulomb friction model in MSC.ADAMS is used for calculating tangential friction force vector $\mathbf{F}_{T}^{i}=\left[\begin{array}{lll}F_{T, x} & F_{T, y} & F_{T, z}\end{array}\right]^{T}$ in Figure 12. Based on this model, the frictional force acting at point I can be expressed as equation (24) $[9,23]$.

$\mathbf{F}_{T}^{i}=\mu\left(\mathbf{V}_{s}\right) \mathbf{F}_{N}^{i}$

$\mu\left(\mathbf{V}_{s}\right)=$ friction coefficient defined as a function of slip velocity vector $\mathbf{V}_{s}$ at contact point I [9]. The contact parameters listed in Table 4 are used for calculating normal and tangential forces. The contact force $\mathbf{F}_{C}^{i}$ on crawler shoe $i$ is the vector sum of normal and frictional forces given by equation (25).

$\mathbf{F}_{C}^{i}=\mathbf{F}_{N}^{i}+\mathbf{F}_{T}^{i}$

The friction torque $\mathbf{T}_{C}^{i}$ about the contact normal axis in Figure 12 impedes any relative rotation of shoe $i$ with respect to the ground. This torque is proportional to the friction force $\mathbf{F}_{T}^{i}$ as given by equation (26) [9].

$\mathbf{T}_{C}^{i}=\frac{2}{3} R \mathbf{F}_{T}^{i}$

$R=$ radius of the contact area. Finally, on crawler shoe 13, the kinematic equations for the two types of driving constraint are specified to simulate propel motion. Figure 13 shows the completed 3-D virtual track-terrain interaction prototype simulator developed in MSC ADAMS. The DAEs generated in the virtual crawler-terrain interaction simulator for the propel motion are integrated over time using GSTIFF integrator with SI2 formulation of ADAMS dynamics solver [9]. The virtual model is simulated for 10secondsto obtain the crawler kinematics for the two types of motion constraints. 
Before propel, the oil sand model, along with crawler track, is allowed to reach its static equilibrium. More detailed formulation for the generalized inertia, external and constraint forces acting on the crawler shoes and oil sands units; analytical verification of MSC ADAMS for solving crawler-terrain dynamics; and the solution methodology used in MSC ADAMS to solve the dynamic equations of motion along with kinematic equations can be found in Frimpong and Thiruvengadam [24].

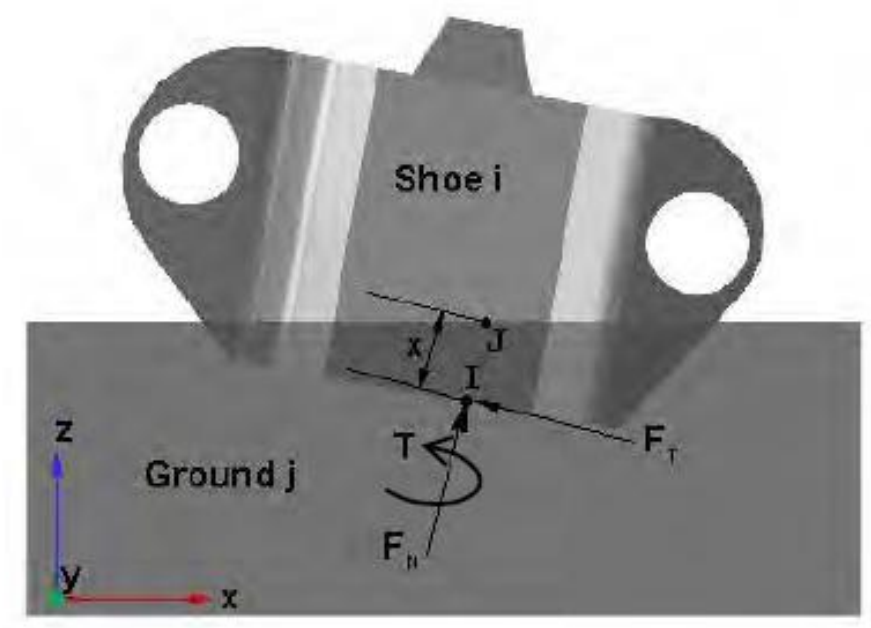

Figure12. Contact forces on crawler shoe, $i$

Table3. Oil sand properties [8, 21]

\begin{tabular}{|c|c|}
\hline Stiffness $(\boldsymbol{k})(\mathbf{M N} / \mathbf{m})$ & Damping $(\boldsymbol{c})(\mathbf{k N}-\mathbf{s} / \mathbf{m})$ \\
\hline 20 & 120 \\
\hline
\end{tabular}

Table4. Contact parameters used in the study [9, 21]

\begin{tabular}{|c|c|c|c|}
\hline \multicolumn{2}{|c|}{ Normal Force } & \multicolumn{2}{c|}{ Friction Force } \\
\hline Contact Stiffness $(k, \mathrm{~N} / \mathrm{m})$ & $1.0 \mathrm{E}+08$ & Static Coefficient $\left(\mu_{\mathrm{s}}\right)$ & 0.4 \\
\hline Max. Contact Damping $\left(c_{\max }, \mathrm{N}-\mathrm{s} / \mathrm{m}\right)$ & $1.0 \mathrm{E}+04$ & Dynamic Coefficient $\left(\mu_{\mathrm{d}}\right)$ & 0.3 \\
\hline Force Exponent $(e)$ & 2.0 & Static transition velocity $\left(\mathrm{V}_{\mathrm{st}}, \mathrm{m} / \mathrm{s}\right)$ & 0.01 \\
\hline Max. Penetration Depth $(d, \mathrm{~m})$ & $1.0 \mathrm{E}-04$ & Dynamic transition velocity $\left(\mathrm{V}_{\mathrm{d}}, \mathrm{m} / \mathrm{s}\right)$ & 0.1 \\
\hline
\end{tabular}

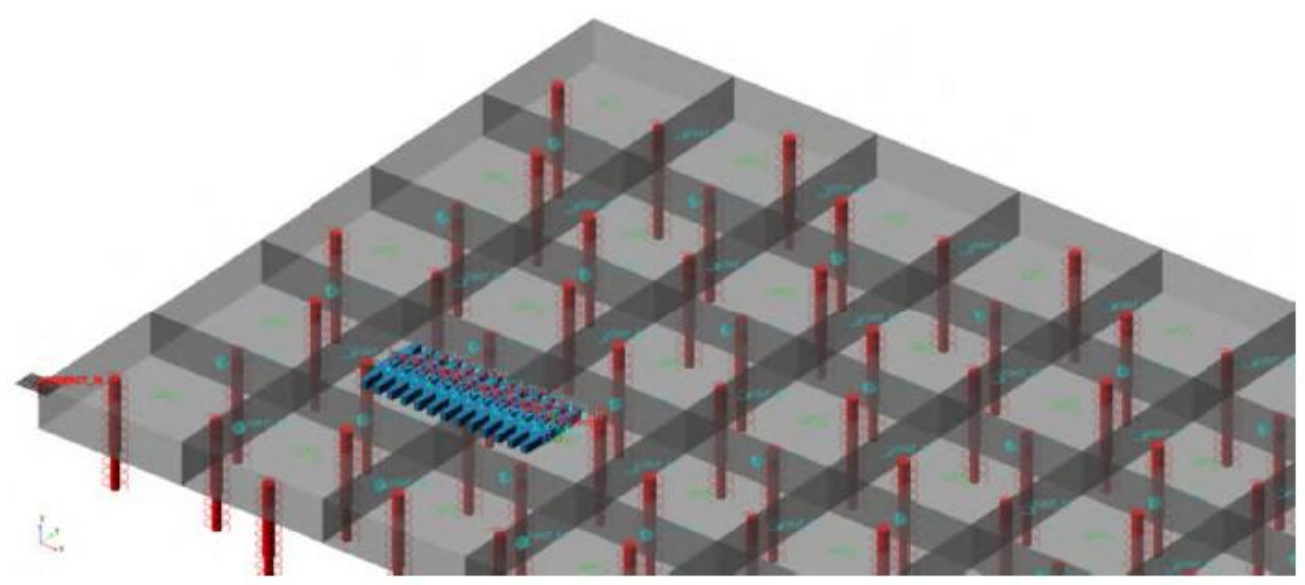

Figure13. $3 D$ rigid virtual crawler track-terrain interactionprototype

\section{KinEMATICS RESUltS}

Case 1 - Translation Only Motion: The displacement of different crawler shoes as a function of time along $\mathrm{x}, \mathrm{y}$ and $\mathrm{z}$ directions is presented for 10 seconds in Figure 14. The displacement of the center of mass of shoe 13 in the global $\mathrm{x}$-direction is obtained analytically from equations (14) and (15). Assuming $t_{1}=0$ and $t_{2}=5 \mathrm{~s}$ in equation (15), the displacement of shoe 13 can be calculated as equation (27).Equation (27) shows that the global x-coordinate of the center of mass of Part 14 (crawler shoe 13) increases non-linearly during the first 5 seconds and then increases linearly during 
the next 5 seconds. From Figure 14a the x-displacement in MSC ADAMS for Part 14 agrees with the analytical solution given in equation (27). From Figure 14a, the driving constraints on shoe 13 also determine the motion of other shoes and hence the entire crawler assembly in the x-direction. The corresponding translation displacement along $\mathrm{y}$ and $\mathrm{z}$ directions is shown in Figures $14 \mathrm{~b}$ and $\mathrm{c}$.

$$
B_{x_{14}}=\left[\begin{array}{l}
B_{0, x_{14}}+\left[0.012 \frac{t^{3}}{3}-0.0016 \frac{t^{4}}{4}\right] ; 0 \leq t \leq 5 s \\
B_{0, x_{14}}+[0.25+0.1 *(t-5)] \quad ; t>5 s
\end{array}\right] m
$$

The displacement of the crawler track is not significant in the y direction and has a maximum variation of $7 \mathrm{~mm}$ from the equilibrium position at time $t=0$ as shown in Figure 14b. The displacement of crawler shoes in the z-direction (Figure 14c) shows large fluctuations from their rest position $(\mathrm{t}=0)$ between-3.6 and $2.3 \mathrm{~cm}$ due to vertical bouncing action of crawler track. Figure $14 \mathrm{c}$ also shows that because crawler shoe 1 (Part 2) in Figure 3 does not have joint constraints at its one end, it shows large fluctuations in $z$-displacement in comparison with other crawler shoes for the entire $10 \mathrm{~s}$ propel motion.

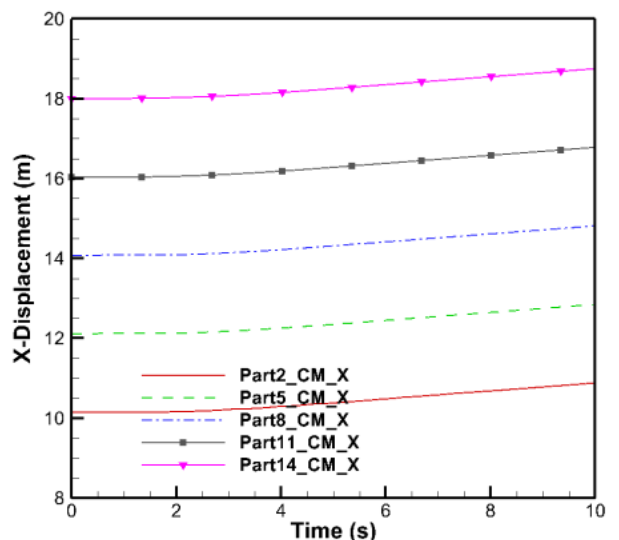

(a)

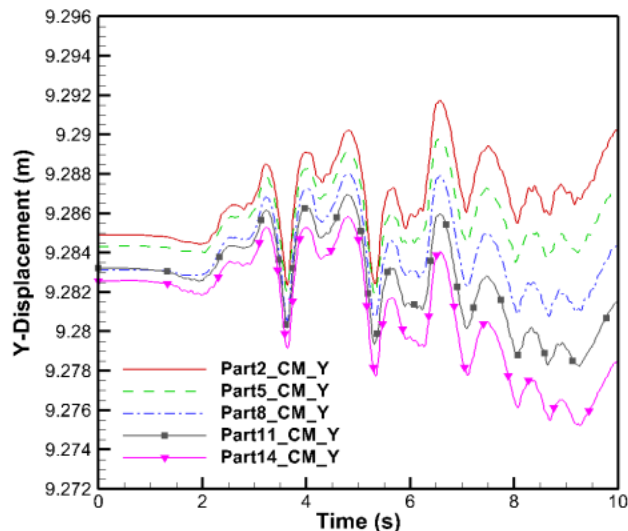

(b)

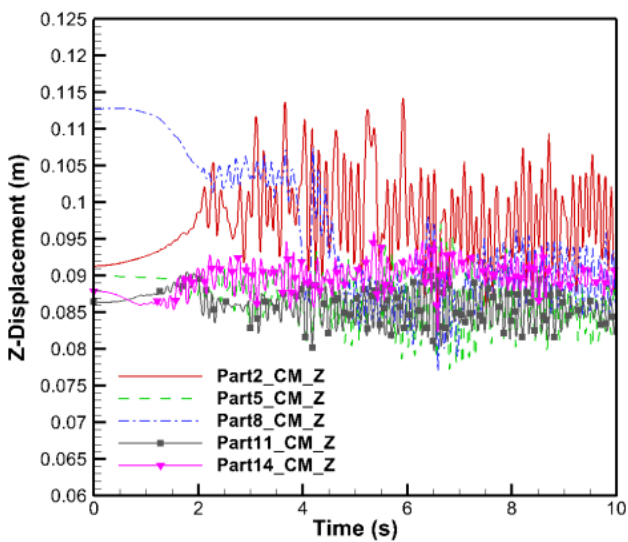

(c)

Figure14. Global $x$, y and zdisplacement of different crawler shoes

The analytical solution for the velocity of Part 14 in the global $x$-direction can be obtained by differentiating equation (27) with respect to time as equation (28).

$\dot{B}_{x_{14}}=\left[\begin{array}{ll}0.012 t^{2}-0.0016 t^{3} ; 0 \leq t \leq 5 s \\ 0.1 & ; t>5 s\end{array}\right] \mathrm{m} / \mathrm{s}$

Equation (28) matches with ADAMS $x$-velocity results for Part 14 plotted as a function of time in Figure 15a. The $\mathrm{x}$-velocity plot in Figure 15 afor Part 14 shows cubic velocity variation during $0 \geq t \leq 5$ $\mathrm{s}$ changing to constant velocity $0.1 \mathrm{~m} / \mathrm{s}$ for $\mathrm{t}>5$ seconds while for other crawler shoes it fluctuates for the entire crawler motion. However, due to propel motion constraint on crawler shoe 13 , the $x$-velocity of all crawler shoes attains an average approximate value of $0.075 \mathrm{~m} / \mathrm{s}$. The time variation of $\mathrm{y}$ velocity in Figure 15b shows that all crawler shoes have approximately same lateral (y) velocity and 
the whole track moves sideways repeatedly with velocity fluctuating between 0.046 and $-0.036 \mathrm{~m} / \mathrm{s}$. Figure $15 \mathrm{c}$ indicates large fluctuations in the $z$-velocity of different crawler shoes with bouncing velocity varying between -0.48 and $0.56 \mathrm{~m} / \mathrm{s}$. Figures $15 \mathrm{~b}$ and $15 \mathrm{c}$ also shows that the lateral $(\mathrm{y})$ and bouncing $(\mathrm{z})$ velocities of different crawler shoes averages to zero.

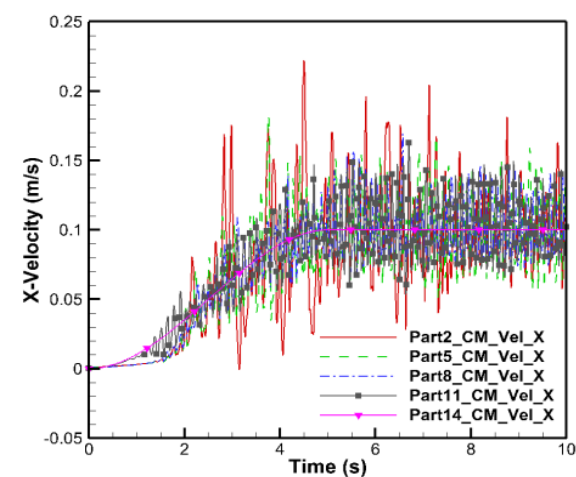

(a)

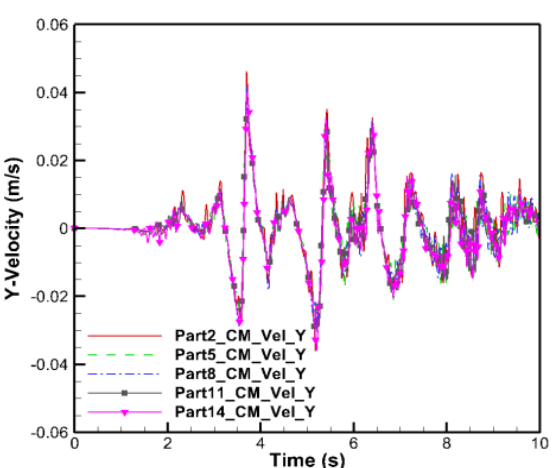

(b)

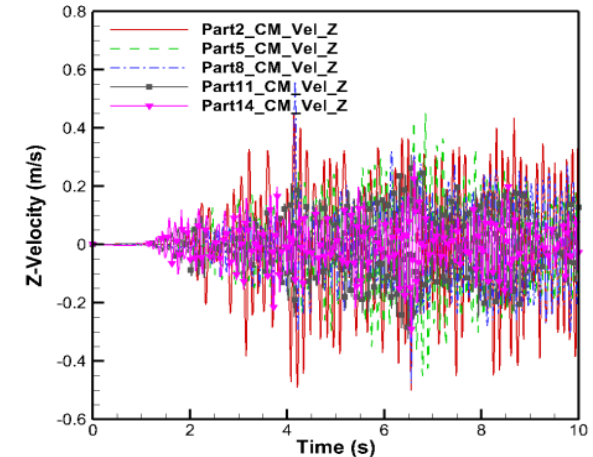

(c)

Figure15. Global $x, y$ and zvelocity of different crawler shoes

Figure 16 shows the time variation of acceleration along longitudinal (x), lateral (y), and vertical (z) directions. The quadratic analytical expression in equation (29) for crawler shoe 13 acceleration along the global $x$-direction agrees with MSC ADAMS results for $\mathrm{x}$-acceleration of Part 14 (crawler shoe $13)$.

$$
\ddot{B}_{x_{14}}=\left[\begin{array}{lc}
0.024 t-0.0048 t^{2} & ; 0 \leq t \leq 5 s \\
0 & ; t>5 s
\end{array}\right] \mathrm{m} / \mathrm{s}^{2}
$$

The maximum acceleration value of $0.03 \mathrm{~m} / \mathrm{s}^{2}$ at $\mathrm{t}=2.5 \mathrm{~s}$ for Part 14 , obtained from equation (29), is negligible in comparison with acceleration of other shoes, which has large spikes and fluctuations ranging between $-7.41 \mathrm{~m} / \mathrm{s}^{2}$ and $4.91 \mathrm{~m} / \mathrm{s}^{2}$ as in Figure 16a. However, all crawler shoes have an average $x$-acceleration value of $0.01 \mathrm{~m} / \mathrm{s}^{2}$. Figures $16 \mathrm{~b}$ and $16 \mathrm{c}$ shows acceleration fluctuating between -1.73 and $1.7 \mathrm{~m} / \mathrm{s}^{2}$ along $y$-direction and between -25.4 and $34.9 \mathrm{~m} / \mathrm{s}^{2}$ along $z$-direction. The lateral $(\mathrm{y})$ acceleration of different crawler shoes, which averages between 0 and $0.006 \mathrm{~m} / \mathrm{s}^{2}$, is negligible in comparison with vertical $(\mathrm{z})$ acceleration average which lies between -0.03 and 0.04 $\mathrm{m} / \mathrm{s}^{2}$, respectively.

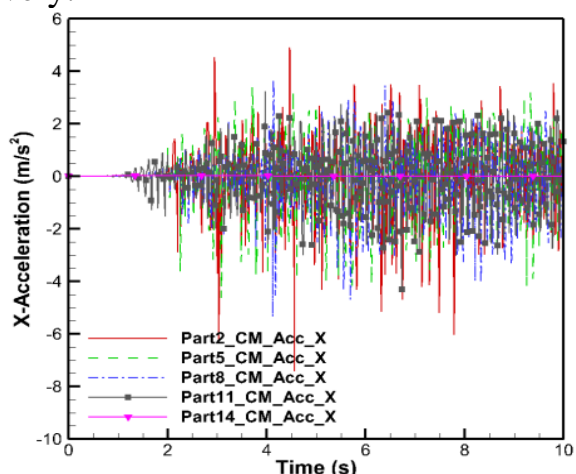

(a)

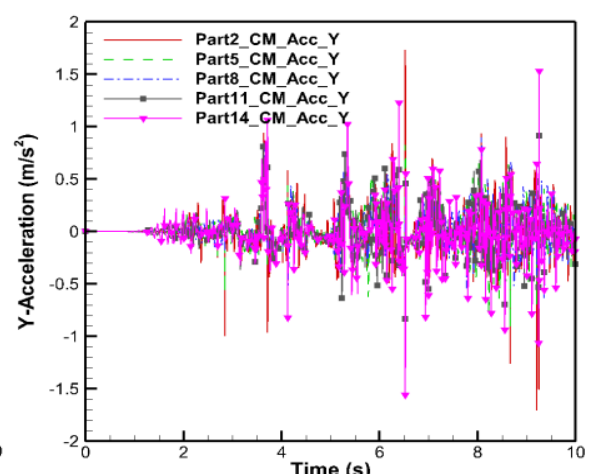

(b) 


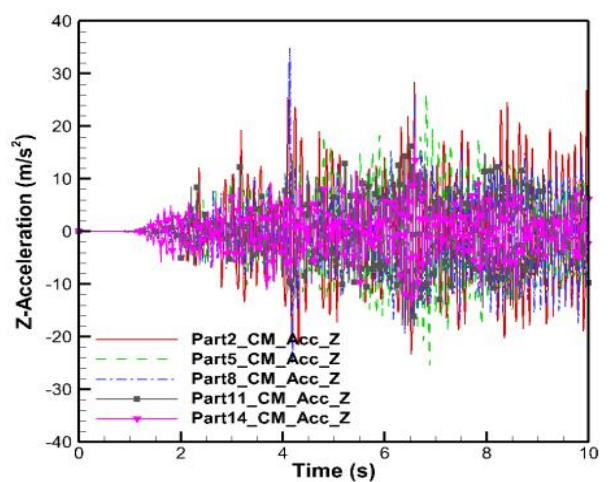

(c)

Figure16. Global $x$, $y$ and zacceleration of different crawler shoes

The angular velocity of different crawler shoes is shown in Figure 17. From Figure 17a, all the crawler shoes have the same angular velocity about the $x$-axis with time and hence the entire crawler track rolls about $x$-axis. Figure 17a also shows that the $x$-angular velocity fluctuates between -9.4 and $7.7 \mathrm{deg} / \mathrm{s}$ with an average value of $-0.09 \mathrm{deg} / \mathrm{s}$ during propel. This average velocity causes a small tilt in the crawler track during propel. The angular velocity about $y$-axis for different shoes exhibit large unsteadiness varying between -133 and $62.2 \mathrm{deg} / \mathrm{s}$ (Figure 17b). Similarly, the angular velocity about $z$-axis (Figure 17c) fluctuates between-3.4 and $1.3 \mathrm{deg} / \mathrm{s}$. Although large fluctuations occur, angular velocity about $y$-axis for different shoes averages between -0.47 and $0.67 \mathrm{deg} / \mathrm{s}$ while about the $z$-axis the mean angular velocity lies between -0.02 and $0.01 \mathrm{deg} / \mathrm{s}$. The angular velocity about $\mathrm{z}$-axis is responsible for repeated rotations of crawler track but due to negligible mean angular velocity about $\mathrm{z}$-axis, the crawler can continue its translation motion along global $\mathrm{x}$-direction.

The angular acceleration variation with time about $x, y$ and $z$ axes for different crawler shoes (Figures $18 \mathrm{a}, 18 \mathrm{~b}$ and $18 \mathrm{c}$ )fluctuates between -208.6 and $228.8 \mathrm{deg} / \mathrm{s}^{2}, 3965.2$ and $6460.8 \mathrm{deg} / \mathrm{s}^{2}$ and -107.1 and $151.7 \mathrm{deg} / \mathrm{s}^{2}$, respectively. The large fluctuation in angular acceleration about y-axis is due to sharps pikes that appear in angular velocities in Figure 17b. Since angular acceleration variation about $\mathrm{x}$-axis is similar for all the crawler shoes (Figure 18a), its average value is around $0.07 \mathrm{deg} / \mathrm{s}^{2}$ while about $y$ and $z$ axes the angular accelerations averages between -8.1 and $23.2 \mathrm{deg} / \mathrm{s}^{2}$ and -0.22 and 0.44 $\mathrm{deg} / \mathrm{s}^{2}$, respectively.

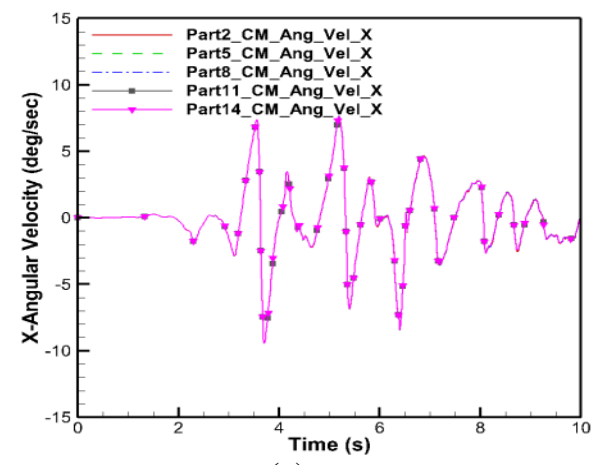

(a)

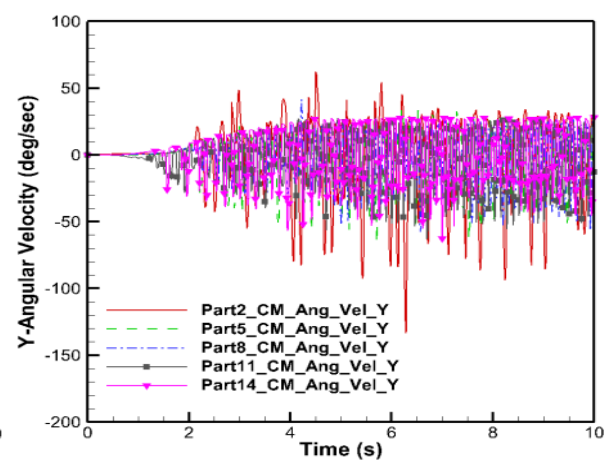

(b)

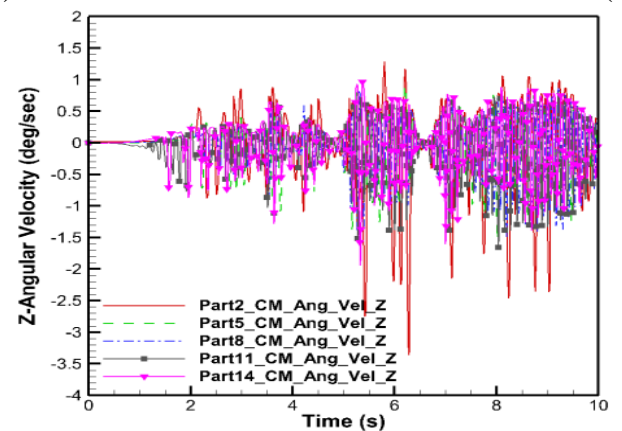

(c)

Figure17. Global $x, y$ and zangular velocity of different crawler shoes 


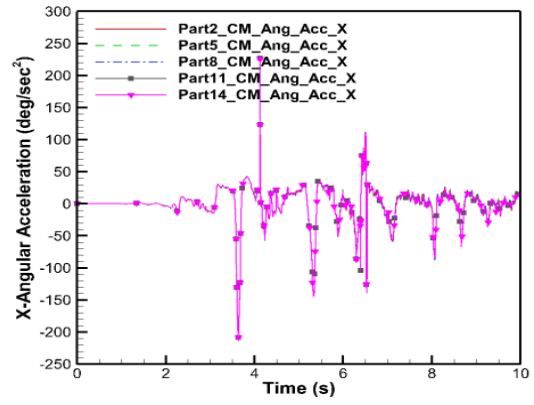

(a)

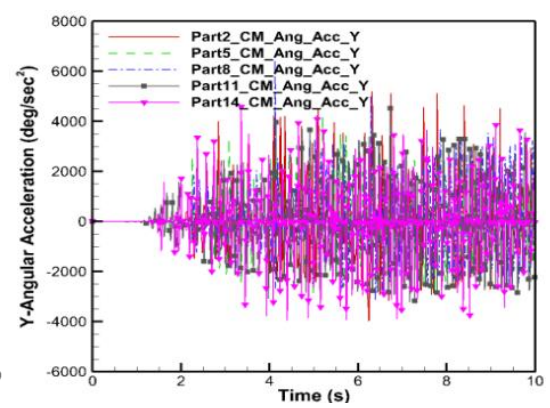

(b)

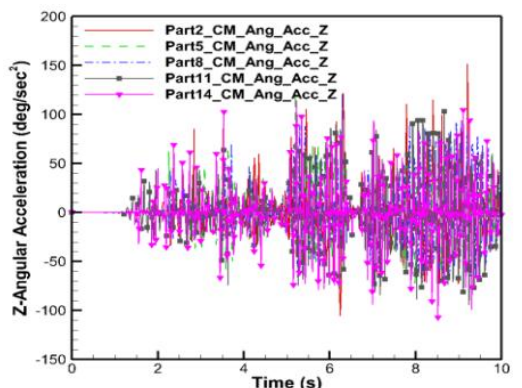

(c)

Figure18. Global $x, y$ and zangular acceleration of different crawler shoes

Case 2 - Translation and Rotation: The variation of displacement, velocity and acceleration as a function of time for Part 14 in the global x-direction follows Figures 14-16 since equations (14) and (15) also applies to case 2 for translation motion. Equation (16)is a path function [12] and analytical expression for Part 14 cannot be obtained for angular velocity and angular acceleration about global zaxis. For case 2, due to space limitations, only numerical results for crawler shoe 10 (Part 11) is plotted and compared with corresponding results from translation-only motion type. Figure 19 shows the center of mass displacement variation of Part 11 with time along the global $\mathrm{x}, \mathrm{y}$ and $\mathrm{z}$ axes. Case 2 follows the same translation equations (14) and (15). Thus, the x-displacement of shoe 10 (Figure 19a)overlaps with each other for both types of motion. The crawler shoe 10 displacement in the global $y$-direction increases during turning in comparison with nearly constant value for translation-only motion as in Figure 19b. This increase in y-displacement is due to crawler track lateral sliding during turning motion. The displacement of Part 11along the $\mathrm{z}$-axis (Figure 19c) shows similar variation for both motion types for $\mathrm{t} \leq 5 \mathrm{~s}$. For $\mathrm{t}>5 \mathrm{~s}$, the $\mathrm{z}$-displacement for turning shows large unsteady behavior as in Figure 19c. The figure shows the maximum $\mathrm{z}$-displacement from equilibrium $(\mathrm{t}=0)$ for turning increases by $8 \%$ when compared with translation-only motion for Part 11 (shoe 10).

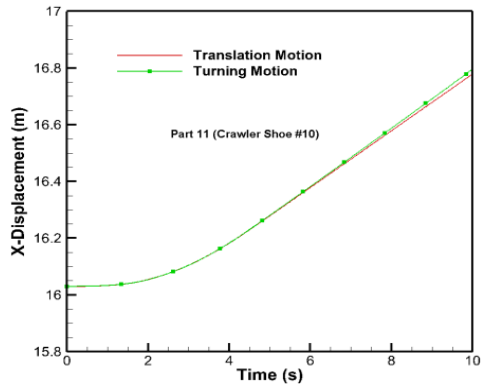

(a)

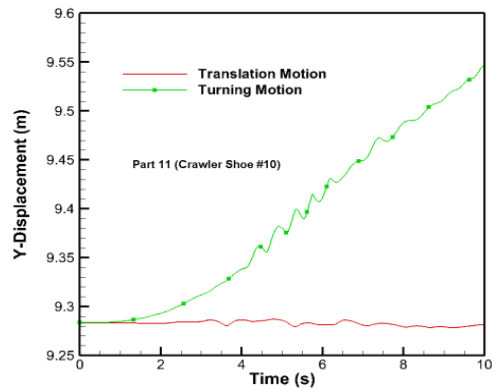

(b)

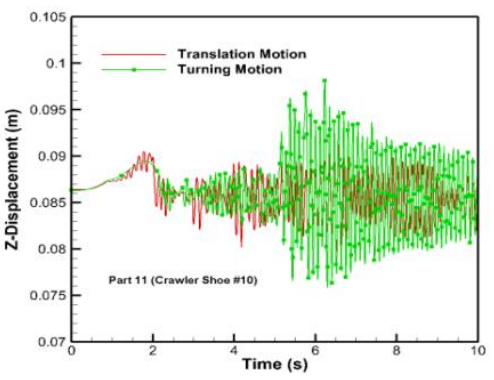

(c)

Figure19. Displacement of crawler shoe 10 in the global $x, y$ and $z$ directions 
Figure 20 shows the comparison of the time variation of velocity of shoe 10 for the two motion types. The $x$-velocity behavior is similar for both motion types as in Figure 20a because of the same translation constraint. However, due to the presence of rotational constraint on crawler shoe 13, the fluctuations in $x$-velocity for turning motion are reduced in comparison with translation-only motion (Figure 20a). The velocity variations with time for Part 11 along $y$ and $z$ directions show large fluctuations for turning motion in comparison with translation motion as in Figures 20b and 20c. The results also show that the maximum $y$-velocity during turning is 5 Xthe maximum value of translation motion while the maximum bouncing velocity ( $z$-velocity) during turning is approximately $1.9 \mathrm{X}$ the maximum value predicted for translation motion.

The acceleration of shoe 10 in the global $x, y$ and $z$ directions during turning exhibits similar behavior as the velocity variation (Figure 20). The comparison of time variation of angular velocity of shoe 10, about the global $x, y$ and $z$ axes, is shown in Figure 21. The rolling behavior of the crawler track has increased during turning in comparison with translation resulting in maximum angular velocity about $\mathrm{x}$-axis during turning to increase by about $4.5 \mathrm{X}$ the maximum value obtained for translation as in Figure 21a. Figure 21b shows similar distributions for angular velocity about $y$-axis for both motion types but with reduced fluctuations during turning when compared with translation. The time variation of $z$-angular velocity of shoe 10 shows fluctuations for both motion types as in Figure 21c. The average $z$-angular velocity value for turning due to rotation motion constraint on Part 14is0.77 $\mathrm{deg} / \mathrm{s}$ when compared with nearly zero average value for translation motion. The comparison of angular acceleration variation of shoe 10 about $x, y$ and $z$ axes for the two motion types also exhibit similar fluctuating characteristics of angular velocity variation shown in Figure 21.

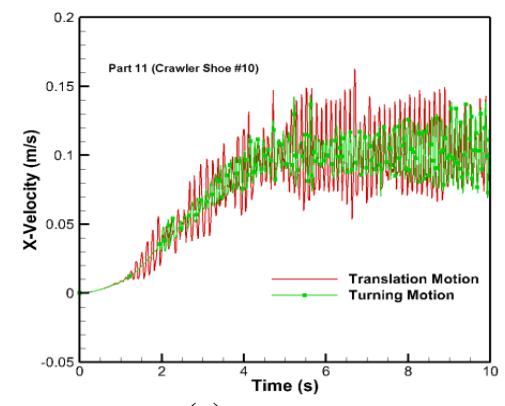

(a)

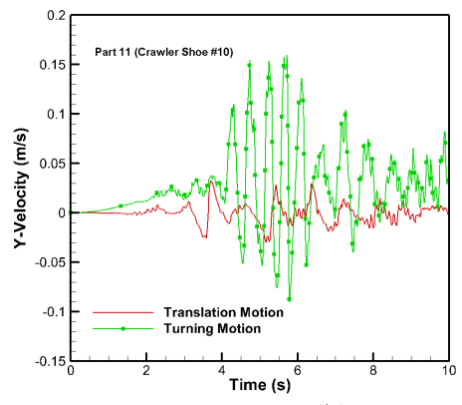

(b)

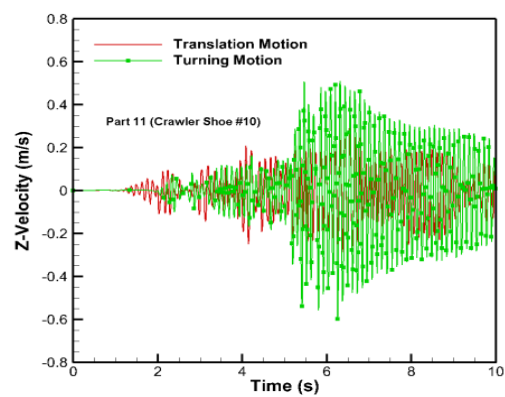

(c)

Figure20. Velocity of crawler shoe 10 in the global $x, y$ and $z$ directions

\section{The VAlue of THE Crawler Kinematic Results}

The shovel propel is achieved through the pulling of the crawler track due to the imposed driving constraint equations at the center of mass of shoe 13. In reality, the crawler tracks are not pulled but rollers roll on the continuous crawler track to cause shovel propel motion. However, pulling or dragging propel motion can still be used for the case when the crawler track slides. This actually occurs in the field when the shovel executes loading duty cycle on soft oil sands terrain or when it makes a turning propel motion. This pulling driving constraint on shoe 13 causes the entire crawler track to slide resulting in dynamic slip velocities at the interface between each track shoe and oil sand. These slip velocities cause tangential or frictional contact forces in the longitudinal and lateral directions on the crawler track. This in turn creates the tractive effort required for propel or turning motions of the shovel $[9,22]$. These tangential forces, along with the normal forces, cause the oil sands terrain to undergo vertical translation and pitching motion. This dynamic terrain deformation dictates the crawler track vertical bouncing, horizontal sliding, rolling and pitching motions and link pin rotation during the shovel duty cycle. 


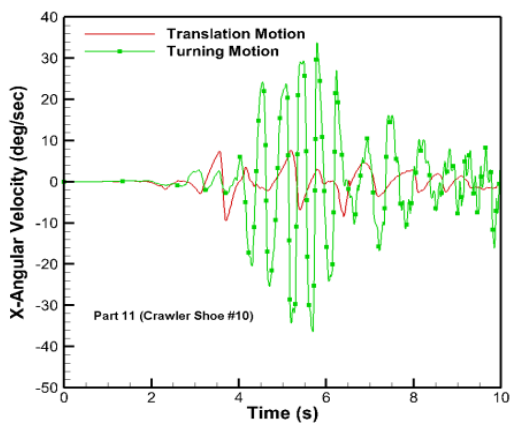

(a)

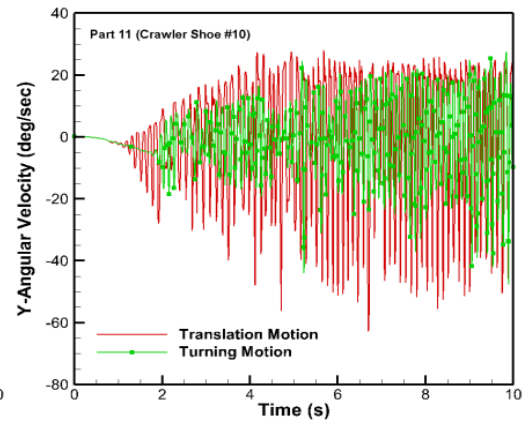

(b)

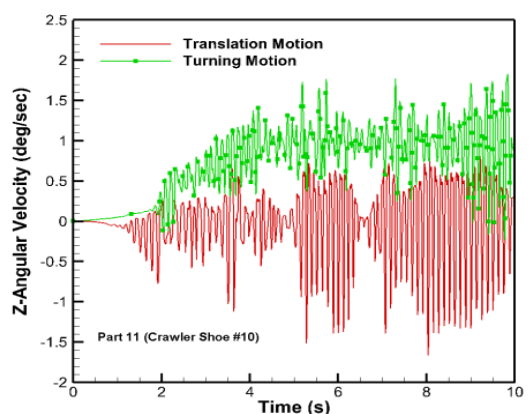

(c)

Figure21. Angular velocity of crawler shoe 10 about global $x, y$ and $z$ axes

In addition, the non-linear nature of the velocity constraint equations for translational and rotational motions simulates the crawler track motion. This motion impacts the magnitude of the initial transients and maximum fluctuations in the contact forces, as well as the time periods for dissipation during the track-terrain interaction. These initial transients and maximum fluctuations in contact forces affect dynamic stress loading in the crawler shoes, which in turn determine the wear, crack and fatigue life of crawler shoes.

\section{CONCLUSiOnS}

Kinematic models have been developed to capture the motions associated with the crawler assembly for the P\&H 4100C BOSS cable shovel in oil sands terrain. The kinematic equations for the crawler shoe joints, oil sands units joints, motion constraints on oil sands units, and two types of driving constraints on crawler track have been established using multi-body dynamics theory to capture crawler-terrain interaction during shovel propel motion. The kinematic equations developed for the crawler track-oil sands assembly resulted in 132 DOFs in the system and the requirement of dynamic analysis to calculate these independent coordinates. The 3-D crawler track-oil sands rigid virtual prototype model is built to simulate crawler-oil sands interaction kinematics for 10 seconds translational and turning motions in MSC.ADAMS. The simulation results for translation driving constraints show that the crawler track propels with a mean velocity of $0.075 \mathrm{~m} / \mathrm{s}$ along $\mathrm{x}$-direction and the mean velocity is close to zero along $y$ and $z$-directions.

Angular velocity fluctuations reach a maximum value of 9.4, 133.0, and $3.4 \mathrm{deg} / \mathrm{s}$ about the global $x, y$ and $z$ axes, while for angular accelerations the fluctuations attain the maximum values of 228.8, 6460.8 and $151.7 \mathrm{deg} / \mathrm{s}^{2}$,about the global $x, y$ and $z$ axes respectively. The mean angular velocity of different crawler shoes about $\mathrm{x}$-axis is $-0.09 \mathrm{deg} / \mathrm{s}$, and about $y$ and $z$-axes, the mean value lies between -0.47 and $0.67 \mathrm{deg} / \mathrm{s}$ and -0.02 and $0.01 \mathrm{deg} / \mathrm{s}$, respectively. Similarly, for angular acceleration of different crawler shoes, the average value about $x$-axis is fixed at $0.07 \mathrm{deg} / \mathrm{s}^{2}$, and the average value lies within- 8.1 and $23.2 \mathrm{deg} / \mathrm{s}^{2}$ and -0.22 and $0.44 \mathrm{deg} / \mathrm{s}^{2}$ about global $y$ and $z$ axes, respectively. During turning motion, the maximum value of $\mathrm{y}$ and $\mathrm{z}$ components of velocity fluctuation attain $5 \mathrm{X}$ and $1.9 \mathrm{X}$ the maximum values obtained for translation motion. The rolling behavior of the crawler track increases during turning due to maximum angular velocity about $\mathrm{X}$-axis being 4.5Xhigher than that for the translation motion. The mean angular velocity about $z$-axis during turning is $0.77 \mathrm{deg} / \mathrm{s}$ causing the crawler track to undergo rotation while for translation motion it is negligible ranging between-0.02 and $0.01 \mathrm{deg} / \mathrm{s}$, keeping the crawler in straight propel motion. The study is a pioneering effort towards understanding shovel crawler performance in harsh and abrasive surface mining terrains. 


\section{REFERENCES}

[1] JGI. 2012. 4100C Boss Electric Mining Shovel - AC Drive Product Overview. (C) Joy Global Inc., Milwaukee, WI. http://www.phmining.com/PHMining/Mining-Equipment/Electric-Shovels/4100C-BOSS .htm

[2] JGI. 2015. Electric Rope Shovels. (C) Joy Global Inc., Milwaukee, WI. http://www.phmining.com/ PH Mining/Mining-Equipment/Electric-Shovels.htm.

[3] P\&H Mining. 2003. Peak Performance Practices: Lower works. (C) P\&H MinePro Services, Milwaukee, WI.

[4] Nakanishi, T. and A.A. Shabana. 1994. Contact forces in the non-linear dynamic anlysis of tracked vehicles. Int. J. for Numerical Methods in Engineering, Vol. 37: 1251-1275.

[5] Choi, J.H., H.C. Lee and A.A. Shabana. 1998. Spatial Dynamics of Multibody Tracked Vehicles. Part I: Spatial Equations of Motion. Vehicle Sys. Dynamics, Vol. 29, No. 1: 27-49.

[6] Rubinstein, D. and R. Hitron. 2004. A Detailed Multi-Body Model for Dynamic Simulation of Off-Road Tracked Vehicles. Journal of Terramechanics, 41: 163-173.

[7] Frimpong, S. 2015. Course Notes on MIN ENG 4933 Surface Mining Methods and Equipment. Missouri University of Science and Technology, Rolla, MO.

[8] Frimpong, S., and Y. Li. 2007. Stress Loading of Cable Shovel Boom under In-situ Digging Conditions. Engineering Failure Analysis 14: 702-715.

[9] MSC. 2013. MSC ADAMS Software. (C) MSC Software Corp. Newport Beach, CA.

[10] Lindberg, D. 2012. MSC ADAMS Modeling of Mechanical System in A400M Crew Entrance Door. Master Thesis, Linkoping Institue of Technology, Sweden.

[11] Shabana, A.A. 2010. Computational Dynamics, $3^{\text {rd }}$ Edition. (C) John Wiley \& Sons, NY.

[12] Shabana, A.A. 1998. Dynamics of Multi-Body Systems, $2^{\text {nd }}$ Ed. (C) Cambridge Uni. Press.

[13] Haug, E.J. 1989. Computer-Aided Kinematics and Dynamics of Mechanical Systems. Allyn and Bacon Series in Engineering. ISBN-13: 978-0205116690; () Prentice Hall.

[14] Ma, Z.D. and N.C. Perkins. 1999. A Hybrid Track Model for Studying Dynamic Track/Sprocket Contact. Proc. of the ASME Noise Control and Acoustics Div.: 147-155.

[15] Madsen, J. 2007. High Fidelity Modeling and Simulation of Tracked Elements for Off-Road Applications using MSC/ADAMS. TR-2007-05," SBE Eng Lab, Univ. of WI, Madison. http://sbel.wisc.edu/ documents /Independent\%20Study\%20Report\%20final\%20edit.pdf

[16] Flores, P., J. Ambrosio and J.P. Claro. 2004. Dynamic Analysis for Planar Multi-Body Mechanical Systems with Lubricated Joints. Multibody System Dynamics, 12: 47-74.

[17] Ravn, P. 1998. A Continous Analysis Method for Planar Multi-Body Systems with Joint Clearance. Multibody System Dynamics, 2: 1-24.

[18] Madsen, J., T. Heyn and D. Negrut. 2010. Methods for Tracked Vehicle System Modeling and Simulation. http://sbel.wisc.edu/Publications/

[19] Flores, P., J. Ambrosio. 2004. Revolute Joints with Clearance in Multi-Body Systems. Computers and Structures, 82: 1359 - 1369.

[20] Schiehlen, W., N. Guse and R. Seifried. 2006. Multibody Dynamics in Computational Mechanics and Engineering Applications. Comp. Meth. Appl. Mech. Eng. 195: 5509-5522.

[21] Frimpong, S., G. Galecki and Y. Li. 2012. Dump Truck Tire Stress Simulation for Extended Service Life. Transactions of SME, Vol. 332: 422-429.

[22] Wong, J.Y. 2001. Theory of Ground Vehicles, $3^{\text {rd }}$ Edition. (C) John Wiley \& Sons, NY.

[23] Frimpong, S., Y. Li and R. Suglo. 2013. Dynamic Torque and Soil Deformation Mechanics and Simulation of the GAP Machinery. Sp. Ed. Surface Mining.J. of Powder Metal. and Mining, Vol. 2, No. 1. ISSN: 2168-9806.

[24] Frimpong, S. and M. Thiruvengadam. 2015. Multi-Body Dynamic Modeling and Simulation of CrawlerFormation Interactions in Surface Mining. Global J. of Research in Engg. (F), Vol. 15, Issue 5: 29-49.

Citation: S. Frimpong, M. Thiruvengadam. (2018). Multi-Body Kinematics of Shovel Crawler Performance in Rugged Terrains, International Journal of Mining Science (IJMS), 4(1), pp.29-45, DOI: http://dx.doi.org/ 10.20431/2454-9460.0401003.

Copyright: (C) 2018 S. Frimpong, M. Thiruvengadam. This is an open-access article distributed under the terms of the Creative Commons Attribution License, which permits unrestricted use, distribution, and reproduction in any medium, provided the original author and source are credited 